\title{
Impaired Skeletal Muscle Function in Patients with Congestive Heart Failure Relationship to Systemic Exercise Performance
}

John R. Minotti, Ian Christoph, Roberta Oka, Michael W. Weiner, Lauren Wells, and Barry M. Massie Cardiology Section and Department of Physical Therapy, San Francisco Veterans Affairs Medical Center, and Department of Medicine and Cardiovascular Research Institute of the University of California, San Francisco, California 94121

\begin{abstract}
In patients with congestive heart failure (CHF), the poor relationship between systemic exercise performance and cardiac function, together with morphologic and metabolic abnormalities in skeletal muscle, raises the possibility that skeletal muscle function may be impaired and limit systemic exercise performance. We assessed strength and endurance of the knee extensors during static and dynamic exercise in 16 patients with Class I-IV CHF and eight age-matched sedentary controls and related these measurements to systemic exercise performance. To assess skeletal muscle function independent of peripheral blood flow, endurance was repeated under ischemic conditions. Strength was not significantly different in the two groups. Dynamic endurance, quantified as the decline in peak torque during 15 successive isokinetic knee extensions, was significantly reduced in the patients compared to controls during aerobic (peak torque $65 \mathrm{vs.} 86 \%$ of initial for exercise at $90 \mathrm{deg} / \mathrm{s}$ and 60 vs. $85 \%$ for exercise at $180 \mathrm{deg} / \mathrm{s} ; P<0.002$ for both), and during ischemic exercise (56 vs. $76 \%$ of initial torque; $P$ $<0.01)$. Static endurance, defined as the time required for force during a sustained maximal voluntary contraction to decline to $60 \%$ of maximal, was reduced in the patients compared to controls (40 \pm 14 vs. $77 \pm 29 \mathrm{~s} ; P<0.02)$. There were highly significant relationships between systemic exercise performance and skeletal muscle endurance at 90 and $180 \mathrm{deg} / \mathrm{s}$ in the patients with $\mathrm{CHF}$ ( $r=0.90$ and 0.66 , respectively). These findings indicate that skeletal muscle endurance is impaired in patients with CHF, that this abnormality is in part independent of limb blood flow, and that these changes may be important determinants of systemic exercise performance. (J. Clin. Invest. 1991. 88:2077-2082.) Key words: congestive heart failure • skeletal muscle function • exercise intolerance $\bullet$ isokinetic exercise
\end{abstract}

\section{Introduction}

Maximal cardiac output limits systemic exercise performance in healthy subjects (1-3). However, in patients with congestive heart failure (CHF), ${ }^{1}$ the mechanism limiting systemic exercise performance is unknown. In CHF patients it is commonly observed that systemic exercise performance correlates poorly

Address correspondence to John R. Minotti, M.D., Section of Cardiology (111C), Veterans Affairs Medical Center, San Francisco, CA 94121.

Received for publication 28 November 1990 and in revised form 14 August 1991.

1. Abbreviations used in this paper: $\mathrm{CHF}$, congestive heart failure.

The Journal of Clinical Investigation, Inc.

Volume 88, December 1991, 2077-2082 with cardiac output, ejection fraction, and left atrial pressures both at rest and during exercise (4-7). The discordance between systemic exercise performance and cardiac function in CHF patients is further demonstrated by the temporal dissociation between the rapid improvement in hemodynamics (within hours after initiating medical treatment) and the gradual improvement in systemic exercise performance (weeks to months after initiating medical treatment) $(8,9)$. These observations suggest that systemic exercise performance in $\mathrm{CHF}$ patients, unlike that in healthy subjects, is not limited solely by central hemodynamics.

A possible factor determining exercise performance in $\mathrm{CHF}$ patients may be impaired skeletal muscle function. Recent work has demonstrated that patients with CHF have abnormalities in skeletal muscle histology and biochemistry (10), and that during exercise their peripheral blood flow (11), and skeletal muscle metabolism $(12,13)$ are abnormal. It is unknown, however, whether skeletal muscle function in CHF patients is impaired, and if so whether impaired function is independent of abnormalities of blood flow. Also, the relationship of skeletal muscle function to systemic exercise performance has not been determined.

The goals of this study were threefold: first, to determine whether skeletal muscle function is abnormal in patients with CHF; second, to determine whether impaired function, if present, is independent of blood flow; and third, to determine whether impaired skeletal muscle function in $\mathrm{CHF}$ patients may be responsible for their exercise limitation.

To measure skeletal muscle function we localized exercise to the knee extensor muscle group, and assessed both strength and endurance during static and isokinetic exercise in CHF patients and age-matched sedentary controls. To determine if impaired endurance is independent of blood flow, we exercised the knee extensor muscles under ischemic conditions and compared muscle endurance in these two groups. To ascertain if skeletal muscle dysfunction could limit systemic exercise performance, we determined the relationship of knee extensor endurance (skeletal muscle function) to peak systemic oxygen consumption (systemic exercise performance).

\section{Methods}

\section{Patient population (Table I)}

16 male subjects with a history of Class I-IV chronic CHF of at least 6 mo in duration were recruited from the Veterans Affairs Medical Center in San Francisco. Salient clinical information concerning these patients is given in Table I. The diagnosis of CHF was based on a history of dyspnea on exertion, fatigue, or fluid retention, with confirmation of left ventricular dysfunction by a radionuclide or echocardiographic ejection fraction of $<40 \%$ (range $7-37 \%$ ). Eight patients had ischemic cardiomyopathy, and eight had primary myocardial disease. Patients were excluded if they had experienced myocardial infarction 
Table I. Physical Characteristics of CHF Patients

\begin{tabular}{lclccc}
\hline Subject & Age & $\begin{array}{c}\text { Cardiac } \\
\text { medication* }\end{array}$ & Peak VO $_{2}$ & $\begin{array}{c}\text { Ejection } \\
\text { fraction }\end{array}$ & NYHA class \\
\hline JH & 57 & D,F,I,H,C & 10 & .17 & 3 \\
BW & 58 & D,F,C & 18 & .32 & 2 \\
BB & 68 & D,F,C & 29 & .30 & 1 \\
AH & 60 & D,F,C & 12 & .35 & 3 \\
KT & 65 & D,F,C,H & 13 & .19 & 3 \\
WG & 78 & D,F,C,H & 08 & .29 & 4 \\
RS & 63 & D,F,C & 17 & .24 & 2 \\
AF & 62 & D,F,C & 12 & .20 & 3 \\
ED & 66 & D,F,C & 16 & .22 & 2 \\
PD & 41 & D,C & 19 & .20 & 4 \\
JL & 71 & D,F,C & 12 & .32 & 1 \\
CD & 48 & D,F,C & 23 & .07 & 2 \\
*BD & 66 & D,F,C & 19 & .37 & 2 \\
*HR & 76 & D,F,C & 14 & .17 & 3 \\
*RH & 70 & D,F,C & 17 & .25 & 3 \\
*CC & 60 & D,H,C & 27 & .26 & 1 \\
Mean & 63 & & 16 & .25 & 2.4 \\
土SD & \pm 9 & & \pm 5 & \pm .08 & \\
\hline
\end{tabular}

D, digoxin; I, isosorbide dinitrate; C, captopril; F, furosemide; H, hydralazine. ${ }^{*}$ Did not participate in static endurance protocol.

within 6 mo or if exercise was limited by symptoms other than fatigue or dyspnea. Other criteria for exclusion included initiation of therapy with an angiotensin converting enzyme inhibitor, long-acting nitrates, diuretics, or digitalis within 3 mo, hemodynamically significant valvular disease, chronic obstructive pulmonary disease, arthritis, or other mechanical limitations to exercise. Patients with peripheral vascular and neurologic disease were also excluded, based upon the presence of normal peripheral pulses and lack of symptoms.

For comparison, eight age-matched sedentary males (mean age of $58 \pm 11 \mathrm{y}$, vs. $61 \pm 9$ for the patients with CHF, $P=N S$ ) were recruited from employees at the Veterans Affairs Medical Center in San Francisco. These subjects had no history of heart disease and no cardiac, peripheral vascular, or muscular abnormalities on physical examination. The protocol was approved by the Committee on Human Research at the University of California, San Francisco, and written informed consent was obtained from all participants.

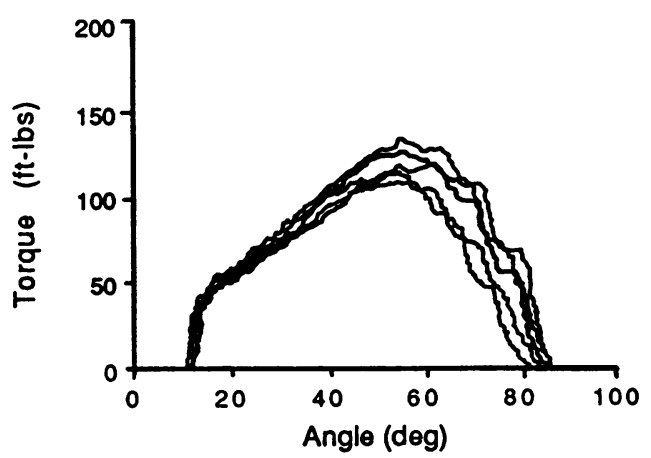

Figure 1. Characteristic torque vs. angle loops for each isokinetic knee extension in a patient with CHF. Each knee extension demonstrates similar initial rates of torque production and similar angles at which maximal torque production was achieved. The consistency of these torque vs. angle loops cannot be achieved with submaximal effort.

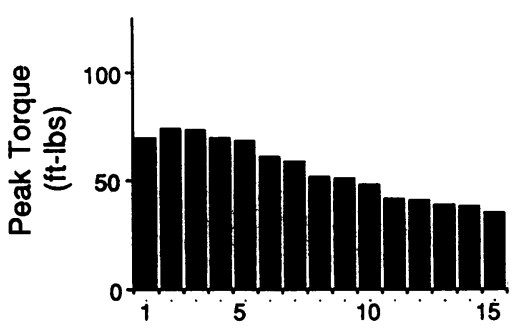

Figure 2. A characteristic record of peak torque output during 15 successive knee extensions is shown for a patient with CHF. In the CHF patients, peak torque declined progressively during exercise. Dynamic endurance is defined as the ratio of the mean peak torque of the last three repetitions as compared to the first three repetitions.

\section{Exercise protocols}

Isokinetic exercise. Dynamic strength and endurance of the knee extensors were measured on an isokinetic dynamometer (340; Cybex, Ronkonkoma, NY), which consists of a fixed length lever arm with its range of motion mechanically limited. The resistance of the lever arm is automatically adjusted in proportion to the dynamic tension produced by the muscle throughout the entire range of motion. This device continuously records torque during isokinetic muscle contraction at a selected angular velocity. Unlike isometric or isotonic muscle testing, the controlled variable during isokinetic contraction is the velocity of the muscle contraction and not the applied resistance.

The subject was stabilized on the adjustable dynamometer testing table. To prevent hip and upper body movement, the backrest was positioned at a 15-deg incline, and a seatbelt and shoulder strap were used to anchor the subject to the backrest. The subject's right knee was then aligned with the input axis of the lever arm, and the ankle was attached to the distal end of the lever arm. After familiarization with the dynamometer, and a standardized warm-up period, each subject was tested at two predetermined velocities ( 90 and $180 \mathrm{deg} / \mathrm{s}$ ) in random order. The exercise protocol consisted of extending the knee 15 times in rapid succession at each velocity. Each knee extension lasted for $\sim 2$-s. The subjects rested for 10-min before exercise at the second velocity. Throughout the procedure, verbal encouragement was given in a standardized manner.

Torque vs. angle (Fig. 1) loops were recorded for each knee extension, to allow determination of the reproducibility of effort. A characteristic record of the torque output during 15 isokinetic knee extensions is shown in Fig. 2. Continuous knee extension resulted in a progressive decrease in torque production throughout exercise in the CHF patients. Dynamic strength was quantified as the mean peak torque produced in the first three knee extensions. Dynamic endurance was defined as the ratio of the mean peak torque in the last three repetitions as compared to the first three repetitions $(14,15)$.

The same knee extension protocol at a velocity of $90 \mathrm{deg} / \mathrm{s}$ was repeated under ischemic conditions in a subset of seven CHF patients and seven controls. A rapid inflating air cuff was secured around the upper thigh and attached to an air source and pressure regulator (D. E. Hokanson Inc., Issaquah, WA). $5 \mathrm{~s}$ before the start of exercise, this cuff was inflated to a pressure of $250 \mathrm{mmHg}$. Absence of blood flow was confirmed at rest and immediately after exercise by strain gauge plethysmography. Each subject performed all 15 knee extensions with the cuff inflated. The patients and the controls denied any discomfort during this exercise protocol, which was completed in $<25 \mathrm{~s}$ by all subjects.

Isometric exercise. Static strength and endurance were measured on the same exercise device. For this portion of the study the lever arm was fixed at $\mathbf{4 5} \mathrm{deg}$ and isometric knee extensions were performed in the following sequence. Three brief (2-3 s) maximal voluntary isometric contractions separated by $60 \mathrm{~s}$ of rest were performed. The highest value was taken as maximal static strength. After a 5-min rest, each subject then performed a sustained maximal voluntary contraction 
while the decline of force was recorded over time. Subjects continued to contract maximally until isometric force declined to $60 \%$ of the initial maximal force. Static endurance time was defined as the time required for isometric force to decline to $60 \%$ of maximal.

Systemic exercise performance. On a separate occasion, systemic exercise was performed using an electronically braked cycle ergometer (Quinton Instruments, Seattle, WA) that maintained constant workload at pedal frequencies of 40-110 rpm. Testing was performed in an air conditioned laboratory with an ambient temperature of $22-24^{\circ} \mathrm{C}$ and humidity of 30-40\%. Before exercise, subjects rested upright on the cycle ergometer for $5 \mathrm{~min}$. Exercise was initially performed unloaded for $2 \mathrm{~min}$. The load was then increased to $200 \mathrm{~kg}-\mathrm{m} / \mathrm{min}(\mathrm{kpm})$ for 2 min, and then by $100 \mathrm{kpm}$ every 2 min until exhaustion. Exhaustion was defined as the inability to maintain a pedal frequency of $>40 \mathrm{rpm}$. Standard verbal encouragement was used for all subjects.

Peak oxygen consumption was measured as an index of systemic exercise performance, and the respiratory quotient was measured to quantify exercise effort. For these measurements, expired gases were collected into a mixing chamber through a mouthpiece and $\mathrm{VO}_{2}$ and $\mathrm{VCO}_{2}$ were measured at 15 -s intervals throughout exercise using a metabolic cart (Sensor Medics Corp., Anaheim, CA). Peak systemic oxygen consumption was defined as the highest oxygen consumption reached during exercise.

\section{Statistical methods}

Differences in muscle endurance, muscle strength, and in peak oxygen consumption between the CHF patients and controls were analyzed by Student's $t$ test for unpaired samples. Linear regression was used to examine the relationships of endurance of the knee extensors with left ventricular ejection fraction and peak systemic oxygen consumption. Statistical significance was assumed for $P$ values $<0.05$ (16). Values are reported as the mean \pm one standard deviation.

\section{Results}

Isometric exercise measurements. Static strength was somewhat, but not significantly lower in the CHF patients than in the normal subjects $(89 \pm 27 \mathrm{ft}-\mathrm{lb}$ vs. $102 \pm 18 \mathrm{ft}-\mathrm{lb}, P<0.15)$. However, the CHF patients fatigued far more rapidly, as indicated by the shorter static endurance time in the 12 patients in whom it was measured $(40 \pm 14$ vs. $77 \pm 29 \mathrm{~s}, P<0.002)$ (Fig. 3$)$.

Isokinetic exercise measurements. Examination of the torque-angle loops (Fig. 1) confirmed that in each subject serial repetitions were performed with a similar initial torque output and achieved peak torque at a similar angle. This degree of reproducibility is consistent with maximum effort. As can be seen from the patient example in Fig. 2, peak torque fell over the 15 repetitions. This decline occurred earlier and was of greater magnitude in the CHF patients.

Dynamic strength, defined as the mean peak torque for the first three isokinetic knee extensions at each velocity tended to be lower in the CHF patients than in the controls both at 90

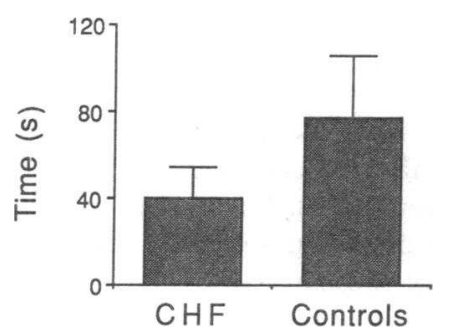

Figure 3. This plot represents the mean \pm 1 SD for isometric endurance, as quantified by the time a maximal voluntary contraction was maintained above $60 \%$ of maximal force in the CHF patients $(n=12)$ and the controls $(n=8)$. The endurance times were markedly lower in the patients with CHF ( $40 \pm 14$ vs. $77 \pm 29 \mathrm{~s}, P<0.002)$.

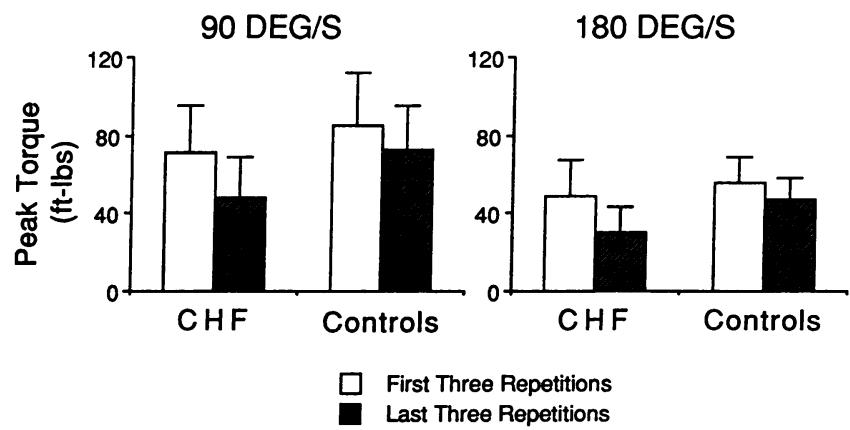

Figure 4. This plot shows the values for peak torque in the initial and final three repetitions in the CHF patients $(n=16)$ and controls $(n$ $=8$ ). At the slower exercise velocity, peak torque declined to $65 \%$ of the initial value in the patients with CHF, which is greater $(P<0.002)$ than the peak torque decline to $86 \%$ of the initial value in the controls. At the faster exercise velocity, peak torque declined to $60 \%$ of the initial value in the patients with CHF, which is greater $(P<0.001)$ than the decline of peak torque to $85 \%$ of the initial value in the controls.

$\mathrm{deg} / \mathrm{s}(68 \pm 22$ vs. $85 \pm 27 \mathrm{ft}-\mathrm{lb}, P=0.12)$, and at $180 \mathrm{deg} / \mathrm{s}$ (46 \pm 16 vs. $56 \pm 13 \mathrm{ft}-\mathrm{lb}, P=0.15$ ) (Fig. 4 ), but these differences did not reach statistical significance.

Dynamic endurance, defined as the ratio of mean torque in the last three isokinetic knee extensions compared to the first three extensions, was reduced in CHF group compared to the control subjects at both exercise velocities. At $90 \mathrm{deg} / \mathrm{s}$, peak torque in the CHF patients declined to $65 \%$ of the initial value (from $68 \pm 22$ to $46 \pm 19 \mathrm{ft}-\mathrm{lb}$ ), which was more $(P<0.002)$ than the decline in peak torque to $86 \%$ of the initial value in the controls (from $85 \pm 27$ to $73 \pm 22 \mathrm{ft}-\mathrm{lb}$ ). The decline in peak torque occurred immediately in the CHF patients, falling to $81 \%$ of its initial value after five repetitions, as compared to 97\% in the controls $(P<0.01)$.

The results at the $180 \mathrm{deg} / \mathrm{s}$ velocity were similar to those at $90 \mathrm{deg} / \mathrm{s}$. Peak torque in the CHF patients declined to $60 \%$ of the initial value (from $47 \pm 16$ to $29 \pm 12 \mathrm{ft}-\mathrm{lb}$ ), which was more $(P<0.001)$ than the decline in peak torque to $85 \%$ of the initial value in the controls (from $56 \pm 13$ to $47 \pm 11 \mathrm{ft}-\mathrm{lb}$ ). Within each group, peak torque declined to similar degrees at the two veloci-

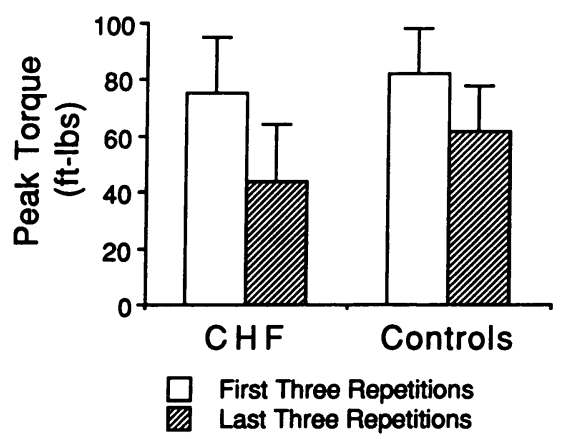

Figure 5. This plot shows the values for peak torque in the initial and final three repetitions in the CHF patients $(n=7)$ and controls $(n$ $=7$ ) during ischemic exercise at the $90 \mathrm{deg} / \mathrm{s}$ velocity. Peak torque declined to $56 \%$ of the initial value in the patients with CHF, which is greater $(P<0.01)$ than the peak torque decline to $76 \%$ of the initial value in the controls. 


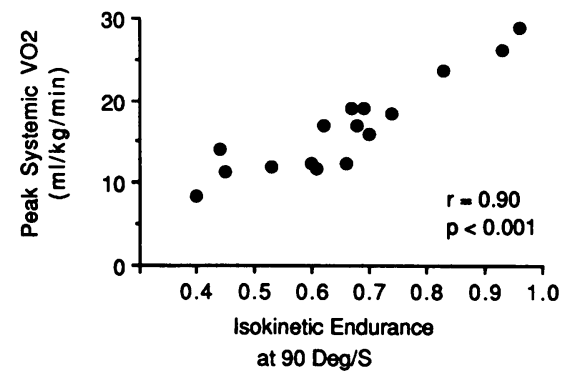

Figure 6. This graph represents the individual values of peak systemic oxygen consumption and dynamic muscle endurance at the $90 \mathrm{deg} / \mathrm{s}$ exercise velocity in the patients with $\mathrm{CHF}(n=16)$. There is a strong correlation between dynamic endurance and peak systemic oxygen consumption $(r=0.90, P<0.001)$.

ties (to $65 \pm 13$ and $60 \pm 13 \%$ in the CHF patients, $P=\mathrm{NS}$, and to $86 \pm 12$ and $85 \pm 10 \%$ in the controls, $P=$ NS) (Fig. 4).

Ischemic isokinetic exercise measurements. During ischemic exercise at $90 \mathrm{deg} / \mathrm{s}$, dynamic endurance was also reduced; peak torque declined further in the patients with $\mathrm{CHF}$ than in the controls. Mean peak torque declined in the CHF patients to $56 \%$ of the initial value (from $75 \pm 21$ to $44 \pm 20$ ), which was more $(P<0.01)$ than the decline in mean peak torque to $76 \%$ of the initial value in the controls (from $82 \pm 14$ to $62 \pm 16$ ) (Fig. 5).

Relationship of muscle endurance to systemic exercise performance. Mean peak systemic oxygen consumption was lower in the CHF patients than in the controls $(16 \pm 5$ vs. $27 \pm 8 \mathrm{ml}$ $\mathrm{O}_{2} / \mathrm{kg} / \mathrm{min}, P<0.05$ ). The relatively low value in the controls confirms their sedentary life style (1), and the range from 8 to $27 \mathrm{ml} \mathrm{O}_{2} / \mathrm{Kg} / \mathrm{min}$ in the CHF patients is consistent with a wide range of functional capacity.

Peak oxygen consumption correlated significantly with dynamic endurance as measured at both velocities in the CHF patients. The correlation was stronger for endurance at $90 \mathrm{deg} /$ s, which is shown in Fig. $6(r=0.90, P<0.001)$, than for endurance at $180 \mathrm{deg} / \mathrm{s}(r=0.66, P=0.005)$. In the control subjects, the correlation between peak oxygen consumption and dynamic endurance was poor both at $90 \mathrm{deg} / \mathrm{s}(r=0.37, P$ $=\mathrm{NS})$ and at $180 \mathrm{deg} / \mathrm{s}(r=0.43, P=\mathrm{NS})$. In both the patients with $\mathrm{CHF}$ and the controls no significant correlations were found for the relationships of peak systemic oxygen consumption vs. either static strength, endurance, or dynamic strength.

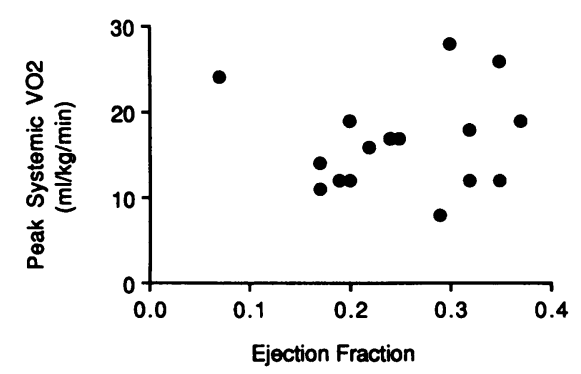

Figure 7. This plot depicts the individual values of peak systemic oxygen consumption and ejection fraction in the CHF patients $(n=16)$. There was no significant correlation between these measurements $(r=0.20)$.
Furthermore, there was no apparent relationship between left ventricular ejection fraction and peak systemic $\mathrm{VO}_{2}$ (Fig. 7).

Heart rate responses during exercise. In the CHF patients during the more strenuous isokinetic protocol at the $90 \mathrm{deg} / \mathrm{s}$ velocity, heart rate rose modestly, by a mean of $13 \mathrm{bpm}$, from $86 \pm 14$ to $99 \pm 15$. The small magnitude of these changes is consistent with the limited muscle mass exercised and with a relatively small cardiovascular stress. The heart rate changes were of lesser magnitude at the $180 \mathrm{deg} / \mathrm{s}$ velocity, from $86 \pm 14$ to $96 \pm 12 \mathrm{bpm}$. During cycle ergometry, heart rate rose to $143 \pm 19 \mathrm{bpm}$.

\section{Discussion}

Exercise intolerance is a frequent symptom in patients with congestive heart failure. Although these patients have impaired cardiac function and hemodynamics, the degree of cardiac dysfunction correlates poorly with their ability to perform systemic exercise. Recent studies have shown morphologic and metabolic abnormalities of skeletal muscle in these patients, suggesting that these changes might result in functional abnormalities in skeletal muscle, and therefore be important in limiting exercise tolerance. In this study, we examined several indices of skeletal muscle function and determined the relationship between muscle function and systemic exercise capacity.

The major finding of this study is that skeletal muscle function is abnormal in CHF. Although muscle strength was relatively preserved, endurance during both static and dynamic exercise was markedly diminished when compared to sedentary age-matched controls. Potential causes for reduced muscle endurance include diminished blood flow to exercising muscle, submaximal effort, and structural or biochemical changes in skeletal muscle.

Zelis and co-workers first described attenuated blood flow responses during forearm exercise and after forearm ischemia in patients with $\mathrm{CHF}$ (11). Although other investigators found no reduction of forearm blood flow in CHF patients who had less fluid retention or lower central venous pressures $(12,17)$, blood flow impairment has been a consistent finding during exercise protocols which provide greater cardiovascular stress and involve larger muscles $(18,19)$. The reduction of peripheral blood flow have been ascribed both to inadequate perfusion pressure and to impaired peripheral vasodilation (11).

The knee extension protocol employed in this study involves a larger muscle group than that exercised in forearm studies, but the relatively brief exercise duration and modest associated hemodynamic changes suggest that muscle performance during this protocol was not limited by cardiac reserve. Indeed, the very rapid decline in peak torque, which achieved statistical significance relative to the controls by the fifth repetition, also supports the hypothesis that an impaired cardiac output response is not the only factor limiting exercise.

To determine definitively whether reduced cardiac output or abnormal muscle blood flow were solely responsible for the diminished muscle endurance, the isokinetic exercise protocol was repeated under ischemic conditions. The finding that abnormal endurance was present during suprasystolic thigh cuff occlusion demonstrates skeletal muscle dysfunction in CHF patients is not due solely to changes in blood flow to exercising 
muscle. This conclusion is also supported by the briefer endurance time found in the patients during prolonged static exercise, because blood flow to muscle is obstructed during maximal sustained isometric contraction at the levels employed in this protocol (20). Whereas these results indicate factors other than inadequate cardiac output and peripheral blood flow are involved in muscle endurance, they do not exclude a role for abnormalities in resting or exercise blood flow in the evolution of muscle dysfunction.

Thus, the reduced muscle endurance in the CHF patients most likely reflects, at least in part, a structural or metabolic defect in skeletal muscle. Muscle atrophy has been demonstrated in patients with $\mathrm{CHF}(21)$ and could play a role in the reduced endurance. However, in preliminary studies by other workers, the degree of atrophy has not correlated well with either exercise capacity or muscle metabolism (22). Also, atrophy is not likely to fully explain the alterations in muscle function observed in the present study, because the changes in endurance were greater than those in muscle strength and the latter usually reflects muscle mass. Furthermore, endurance was quantified relative to the initial torque values, so that generalized muscle atrophy should not have been a major determinant of endurance.

Our findings contrast with the results of Lipkin et al. (10), who found lower maximal static strength in the quadriceps femoris muscle in eight patients with severe CHF than in controls. This difference may result from the wider range of clinical symptoms and exercise capacity in our patients than in the group examined by Lipkin et al. or from their use of more active control subjects. Their patients may have had more muscle atrophy, which would be expected to be associated with reduced strength.

The impaired muscle endurance is consistent with the metabolic changes commonly observed in exercising skeletal muscle in patients with CHF. A rapid accumulation of metabolic byproducts in exercising muscle of CHF patients could be responsible for the decline in muscle contractile force. For example, muscular fatigue has been attributed to intracellular lactate accumulation (23), acidosis, or the increased concentration of inorganic phosphate or its diproteinated form $\left(\mathrm{H}_{2} \mathrm{PO}_{4}^{-}\right)$(2426). Metabolic studies employing magnetic resonance spectroscopy have revealed abnormalities of muscle metabolism in CHF patients $(12,17)$. These studies demonstrated a lower intracellular $\mathrm{pH}$ and faster accumulation of both inorganic phosphate and $\mathrm{H}_{2} \mathrm{PO}_{4}$ at similar workloads in $\mathrm{CHF}$ patients compared to healthy controls, which might then explain the observed reduction in endurance.

Although submaximal effort in these patients cannot be totally excluded as a reason for the decreased muscle endurance, this explanation is unlikely. The torque vs. angle loops recorded during the serial knee extensions demonstrate similar initial rates of torque production and similar angles at which maximal torque was achieved. This consistency of performance cannot be accomplished with submaximal effort. In addition, changes in central command cannot be excluded as a potential cause for the difference in endurance time during static exercise between the groups.

The potential importance of skeletal muscle dysfunction in CHF patients is demonstrated by the significant correlations between isokinetic endurance at both the 90 and $180 \mathrm{deg} / \mathrm{s}$ velocities and peak systemic $\mathrm{VO}_{2}$. This suggests that peripheral skeletal muscle dysfunction might be an important determinant of exercise performance and fatigue. This relationship was particularly close at the slower velocity, which provides a greater resistance and thus involves a greater proportion of both oxidative and glycolytic fibers. The lack of correlation between static endurance and peak $\mathrm{VO}_{2}$ is not surprising, because static, anaerobic exercise is physiologically very different from dynamic aerobic exercise during cycle ergometry.

The strong correlation between muscle function and systemic exercise performance is particularly significant in view of the weak correlation between cardiac function and peak $\mathrm{VO}_{2}$ observed in the present study and many previous investigations (4-7). This disparity between central hemodynamics and exercise performance suggests that impaired muscle endurance is an important determinant of systemic exercise in patients with CHF.

In summary, the major findings of this study are that CHF patients have impaired muscle endurance, which is, in part independent of muscle blood flow. Also, the muscle dysfunction in CHF may have a major role in limiting systemic exercise performance. These results are consistent with the findings that an exercise regimen which stimulates training adaptations in skeletal muscle, is effective in increasing exercise performance in patients with CHF without altering cardiac function or exercise blood flow $(19,27)$ and, conversely, they are consistent with the observation that treatments which rapidly improve central hemodynamics are not paralleled by rapid increases in systemic exercise performance (9).

\section{Acknowledgments}

The authors wish to thank Mimi Zeiger for editorial assistance in preparing this manuscript.

This work was supported in part by the Research Service of the Department of Veterans Affairs, the California Affiliate of the American Heart Association, and grants No. HL 25847 and R01DK33293 of the National Institutes of Health.

\section{References}

1. Mitchell, J. H., and G. Blomqvist. 1971. Maximal oxygen uptake. N. Engl. J. Med. 284:1018-1022.

2. Mitchell, J. H., B. J. Sproule, and C. B. Chapman. 1958. The physiological meaning of the maximal oxygen intake test. J. Clin. Invest. 37:538-547.

3. Wahren, J., and L. Jorfeldt. 1973. Determination of leg blood flow during exercise in man: an indicator-dilution technique based on femoral venous dye infusion. Clin. Sci. Mol. Med. 45:135-146.

4. Lipkin, D. P., and P. A. Poole-Wilson. 1986. Symptoms limiting exercise in chronic heart failure. Br. Med. J. 292:1030-1031.

5. Higgenbothen, M. B., K. G. Morris, E. H. Conn, R. E. Coleman, and F. R. Cobb. 1983. Determinants of exercise performance among patients with congestive heart failure. Am. J. Cardiol. 51:52-60.

6. Franciosa, J. A., S. Ziesche, and M. Wilen. 1979. Functional capacity of patients with chronic left ventricular failure: relationship of bicycle exercise performance to clinical and hemodynamic characterization. Am. J. Med. 67:460466.

7. Szlachcic, J., B. M. Massie, B. L. Kramer, N. Topic, and J. Tubau. 1985. Correlates and prognostic implication of exercise capacity in chronic congestive heart failure. Am. J. Cardiol. 55:1037-1042.

8. Wilson, J. R., and N. Ferraro. 1983. Exercise intolerance in patients with congestive heart failure: relationship to oxygen transport and ventilatory abnormalities. Am. J. Cardiol. 51:1358-1363.

9. Wilson, J. R., J. L. Martin, and N. Ferraro. 1984. Impaired skeletal muscle nutritive flow during exercise in patients with congestive heart failure: role of cardiac pump dysfunction as determined by the effect of dobutamine. Am. J. Cardiol. 53:1308.

10. Lipkin, D. P., D. A. Jones, J. M. Round, and P. A. Poole-Wilson. 1988. 
Abnormalities of skeletal muscle in patients with chronic heart failure. Int. J. Cardiol. 18:187-195.

11. Zelis, R., J. Longhurst, R. J. Capone, and D. T. Mason. 1974. A comparison of regional blood flow and oxygen utilization during dynamic forearm exercise in normal subjects and patients with congestive heart failure. Circulation. 50:137-143.

12. Weiner, D. H., L. I. Fink, J. Maris, R. A. Jones, B. Chance, and J. R. Wilson. 1986. Abnormal skeletal muscle bioenergetics during exercise in patients with heart failure: role of reduced muscle flow. Circulation. 73:1127-1136.

13. Wilson, J. R., L. Fink, J. Maris, N. Ferraro, J. Power-Vanwart, S. Eleff, and B. Chance. 1985. Evaluation of energy metabolism in skeletal muscle of patients with heart failure with gated phosphorus-31 nuclear magnetic resonance. Circulation. 71:57-62.

14. Thorstensson, A., G. Grimby, and J. Karlsson. 1976. Force-velocity relations and fiber composition in human knee extensor muscles. J. Appl. Physiol. 40:12-16.

15. Larsson, L., and J. Karlsson. 1978. Isometric and dynamic endurance as a function of age and skeletal muscle characteristics. Acta Physiol. Scand. 104:129 136.

16. Glantz, S. A. 1987. Primer of Biostatics. McGraw Hill Book Co., New York. 64-77, 195-212.

17. Massie, B. M., M. Conway, R. Yonge, S. Frostick, J. Ledingham, P Sleight, G. Radda, and B. Rajagopalan. 1987. Skeletal muscle metabolism in patients with congestive heart failure. Relation to clinical severity and blood flow. Circulation. 76:1009-1014.

18. LeJemtel, T. H., C. S. Maskin, D. Lucido, and B. J. Chadwick. 1986 Failure to augment maximal limb blood flow in response to one-leg exercise in patients with severe heart failure. Circulation. 74:245-251.
19. Sullivan, M. J., M. B. Higginbotham, and F. R. Cobb. 1988. Exercise training in patients with severe left ventricular dysfunction. Hemodynamic and metabolic effects. Circulation. 78:506-515.

20. Bancroft, H., and J. L. K. Miller. The blood flow through muscle during sustained contraction. 1939. J. Physiol. (Lond.). 97:17-31.

21. Mancini, D. M., D. Nazzaro, L. Georgopoulous, N. Wagner, J. Mullen, and J. R. Wilson. 1991. Skeletal muscle atrophy contributes to exercise intolerance in heart failure. J. Am. Coll. Cardiol. 17:88A. (Abstr.)

22. Mancini, D. M., A. Rein, G. Walter, N. Reichek, R. Lenkinski, and J. R. Wilson. 1991. Skeletal muscle metabolic abnormalities in congestive heart failure are not due to muscle atrophy. J. Am. Coll. Cardiol. 17:158A. (Abstr.)

23. Tesch, P., B. Sjodin, A. Thorstensson, and J. Karisson. 1978. Muscle fatigue and its relation to lactate accumulation and LDH activity in man. Acto Physiol. Scand. 103:413-420.

24. Sahlin, K., L. Edstrom, H. Sjoholm, and E. Hultman. 1981. Effects of lactic acid accumulation and ATP decrease on muscle tension and relaxation. Am. J. Physiol. 240:C121-C126.

25. Sahlin, K., L. Edstrom, and H. Sjoholm. 1983. Fatigue and phosphocreatine depletion during carbon dioxide-induced acidosis in rat muscle. Am. J. Physiol. 245:C15-C20.

26. Miller, R. G., M. D. Boska, R. S. Moussavi, P. J. Carson, and M. W. Weiner. 1988. ${ }^{31} \mathrm{P}$ Nuclear magnetic resonance studies of high energy phosphates and pH in human muscle fatigue. J. Clin. Invest. 81:1190-1196.

27. Minotti, J. R., E. C. Johnson, T. L. Hudson, G. Zuroske, E. Fukushima T. G. Cagle, T. W. Chick, B. M. Massie, and M. V. Icenogle. 1990. Skeleta muscle response to exercise training in congestive heart failure. J. Clin. Invest. 86:751-758. 